TPI-MINN-00/25

UMN-TH-1905/00

PNPI-TH-2396/00

ITEP-TH- $85 / 00$

hep-th/0012250

\title{
Type I Superconductivity upon Monopole Condensation in Seiberg-Witten Theory
}

\author{
A. Vainshtein ${ }^{a}$ and A. Yung ${ }^{a, b, c}$ \\ ${ }^{a}$ Theoretical Physics Institute, University of Minnesota, Minneapolis, MN 55455 \\ ${ }^{b}$ Petersburg Nuclear Physics Institute, Gatchina, St. Petersburg, 188350 用 \\ ${ }^{c}$ Institute of Experimental and Theoretical Physics, Moscow, 117259
}

\begin{abstract}
We study the confinement scenario in $\mathcal{N}=2$ supersymmetric $\mathrm{SU}(2)$ gauge theory near the monopole point upon breaking of $\mathcal{N}=2$ supersymmetry by the adjoint matter mass term. We confirm claims made previously that the Abrikosov-Nielsen-Olesen string near the monopole point fails to be a BPS state once next-to-leading corrections in the adjoint mass parameter taken into account. Our results shows that type I superconductivity arises upon monopole condensation. This conclusion allows us to make qualitative predictions on the structure of the hadron mass spectrum near the monopole point.
\end{abstract}

December 2000

*Permanent address 


\section{Introduction}

According to Mandelstam and 't Hooft ideas [1] confinement of charges appears as a dual Meissner effect upon condensation of monopoles. Once monopoles condense the electric flux is confined in the (dual) Abrikosov-Nielsen-Olesen (ANO) vortex [2] connecting the heavy trial charge and anti-charge. The vortex has a constant energy per unit length (the string tension $T$ ). This ensures that the confining potential between the heavy charge and anti-charge increases linearly with their separation. However, since dynamics of monopoles is hard to control in non-supersymmetric gauge theories, this picture of confinement remained an unjustified qualitative scheme for many years.

The breakthrough in this direction was made by Seiberg and Witten in [3, 4]. Constructing the exact solution of the $\mathcal{N}=2$ supersymmetric gauge theory they showed that the condensation of monopoles really occurs near the monopole point on the moduli space of the theory once $\mathcal{N}=2$ supersymmetry is broken down to $\mathcal{N}=1$ by the mass term of the adjoint matter [3].

More specifically, they considered the $\mathcal{N}=2$ Yang-Mills theory with $\mathrm{SU}(2)$ as a gauge group [3]. The gauge symmetry is broken down to $\mathrm{U}(1)$ by the vev $\langle\varphi\rangle=a \tau_{3} / 2$ of the adjoint scalar field $\varphi$. The complex parameter $a$ is the modulus on Coulomb branch of the theory. Near the monopole singularity on the Coulomb branch (the point where monopoles become massless) the effective low energy theory is the dual $\mathcal{N}=2$ QED. This means that the theory has light monopole hypermultiplet coupled to dual photon multiplet in the same way as ordinary charges are coupled to the ordinary photon.

The $\mathcal{N}=2$ supersymmetry is broken down to $\mathcal{N}=1$ by adding a mass term $\mu \operatorname{Tr} \Phi^{2}$ for the adjoint matter ( $\Phi$ is the adjoint chiral superfield; its scalar component $\varphi$ develops the vev discussed above). After the breaking the whole Coulomb branch shrinks to two points at which either the monopole or dyon become massless [3] (we give a brief review of the phenomenon in the next section). Consider, say, the monopole point. At this point the monopole condensate develops, its magnitude is proportional to the small parameter $\mu$. The monopole condensation ensures $\mathrm{U}(1)$ confinement of trial heavy charges.

Since the work of Seiberg and Witten [3] it was quite important to understand to what extent this $\mathrm{U}(1)$ confinement of electric charges is similar to confinement of color we expect (but cannot control) in QCD. Moreover, we expect the QCD-like confinement also in $\mathcal{N}=1$ supersymmetric QCD which can be obtained as a large $\mu$ limit of the theory under consideration. Unfortunately, we have no control on the dynamics of the theory in this limit (with exception of values of various chiral condensates which are known exactly at any $\mu$ 5 7]).

One distinction noticed by Douglas and Shenker [8] appears in $\mathrm{SU}\left(N_{c}\right)$ theories at $N_{c} \geq 3$. Since $\mathrm{SU}\left(N_{c}\right)$ gauge group is broken down to $\mathrm{U}(1)^{N_{c}-1}$ by the vevs of adjoint

scalars there are $N_{c}-1$ winding numbers, one per each $\mathrm{U}(1)$ factor. Let us remind that the winding number $n=0, \pm 1, \pm 2, \ldots$ counts the flux of 'magnetic' field in the 
ANO vortex (it is an element of $\pi_{1}(\mathrm{U}(1))=\mathbf{Z}$ ). Numerous winding numbers lead to existence of too many hadronic states in the spectrum [8] (see also [9] for the brane interpretation of this result). Namely, the number of distinguishable families of $\bar{q} q$ meson states produced by $p$-string $/(p-1)$-antistring pairs (i.e. objects of the type $\left.\left[n_{i}\right]=[0, \ldots, 0,-1,1,0 \ldots, 0]\right)$ is the integer part of $\left(N_{c}+1\right) / 2$.

Another distinction, visible already in the $\mathrm{SU}(2)$ theory [10], is related with higher winding numbers, $n>1$. This also produces an extra multiplicity in the hadron spectrum if strings with higher winding numbers exist. In QCD or in the large $\mu$ limit of the present theory we expect classification of states under the center of the gauge group, $\mathbf{Z}_{2}$ for $\mathrm{SU}(2)$, rather than $\mathbf{Z}$.

Consider as an example the ANO vortex with two units of the flux, $n=2$. This string connects two quarks with two antiquarks producing an "exotic" state in the spectrum of the theory at small $\mu$. Note, that the string with $n=2$, in principle, can be torn up by $W$ boson pair creation. It does not happen while energy is less than $2 m_{W} \sim \Lambda$ which is a large quantity for $\mu \ll \Lambda$ (here $\Lambda$ is the scale parameter of $\mathrm{SU}(2)$ theory). We do not expect such exotic states to appear in QCD.

The discussion above of "exotic" states in the hadron spectrum [10] is based on the purely topological reasoning. Here we consider the dynamical side of the problem. In fact, strings with multiple $n$ are stable or unstable depending on the type of the superconductor. Namely, in the type I superconductor the tension $T_{n}$ of the vortex with winding number $n$ is less than $n T_{1}$ which is sum of tensions of $n$ separated vortices with the unit flux. Therefore vortices with $n>1$ are stable and we really have a tower of "exotic" states in the spectrum.

On the contrary, in the type II superconductor vortices with $n_{0}>1$ are unstable against decay into $n$ vortices with the unit winding number. Therefore, in this case "exotic" states are unstable and actually in the "real world" at strong coupling might not be observable at all.

The purpose of this paper is to find out the type of superconductivity at the monopole point in the $\mathrm{SU}(2)$ Seiberg-Witten theory perturbed by a small adjoint mass $\mu$. The result will allow us to make a prediction about the presence of an "exotic" state in the hadron spectrum of the theory associated with multiple winding numbers of ANO vortices.

Note, that in this paper we consider the $\mathrm{SU}(2)$ gauge theory and discuss higher winding numbers $|n|>1$ in the single $\mathrm{U}(1)$ group. In Refs. [8, 9] the $\mathrm{SU}\left(N_{c}\right)$ gauge group is considered and $p$-strings with winding numbers $\left|n_{p}\right|=1$ in $p$-th $\mathrm{U}(1)$ factor are studied. The string tensions are shown to be proportional to $\sin \left(\pi p / N_{c}\right)$ which is interpreted as a type I behavior of the $p$-string (see also the second paper in Ref. [11] for a study of a more general deformation of $\mathcal{N}=2$ theory).

It was already noticed [9] that the mass term for the adjoint matter acts as a generalized Fayet-Iliopoulos [12 term to leading order in $\mu$ (see the next section for a 
brief review). To this order, although $\mu \neq 0$, the extended $\mathcal{N}=2$ supersymmetry is preserved in the effective low energy description near the monopole point. Then the superconductivity at the monopole point looks as being on the border between types I and II and the ANO string looks like BPS-saturated [13]. However, in [9] it was conjectured the BPS condition is spoiled by higher orders in the breaking parameter $\mu / \Lambda$. In [11] authors came to the same conclusion.

In this paper we start by studying the effective theory in the vicinity of the monopole point. In leading order which accounts for linear in deviation terms we explicitly demonstrate that $\mathcal{N}=2$ supersymmetry is preserved. This preservation was firstly derived in Ref. [9] within the brane construction. Our consideration shows that together with the preservation of $\mathcal{N}=2$ supersymmetry a certain $\mathrm{U}(1)$ flavor symmetry is also preserved in the same order. In terms of the microscopic $\mathcal{N}=1$ theory it is the $R$ symmetry broken only by quantum anomalies. In the effective theory an anomalous nature of this $\mathrm{U}(1)$ shows up only in 'nonperturbative' corrections of order $\sqrt{\mu / \Lambda}$.

Next we study these corrections. Taken them into account we show that $\mathcal{N}=2$ SUSY is broken to $\mathcal{N}=1$ and superconductivity at the monopole point turns out to be of type I. This result shows that the hadron spectrum of the theory looks very different from what we expect in QCD. As we explained above this means that the tower of "exotic" states with multiple fluxes is present in the hadron spectrum.

The paper is organized as follows. In Sec. 2 we review the confinement scenario near the monopole point. In Sec. 3 we consider the leading order perturbation in $\mu$ and show that $\mathcal{N}=2$ supersymmetry remains unbroken to this order. In Sec. 5 and Sec. 6 we consider next-to-leading order corrections in $\mu / \Lambda$. Sec. 7 contains our conclusions.

\section{Monopole condensation}

In this section we present a brief review on the monopole condensation in the broken $\mathcal{N}=2$ gauge theory.

In the $\mathcal{N}=1$ superfield notations the Seiberg-Witten solution leads to the following effective Lagrangian for the dual $\mathrm{U}(1)$ gauge field $V_{D}(x, \theta, \bar{\theta})$ and its $\mathcal{N}=2$ partner $A_{D}(x, \theta)$,

$$
\mathcal{L}_{\text {eff }}=\frac{1}{8 \pi} \operatorname{Im} \int \mathrm{d}^{2} \theta \tau_{D}\left(A_{D}\right) W_{D}^{2}-\frac{1}{4 \pi} \operatorname{Im} \int \mathrm{d}^{2} \theta \mathrm{d}^{2} \bar{\theta} \bar{A}_{D} A\left(A_{D}\right) .
$$

The solution is parametrized by vevs $a(u)=\langle A\rangle, a_{D}(u)=\left\langle A_{D}\right\rangle$ as functions of the modulus $u=\left\langle\operatorname{Tr} \Phi^{2}\right\rangle$ where $\Phi$ is the the superfield describing the adjoint matter in the original microscopic $\mathrm{SU}(2)$ theory. In terms of these functions

$$
\tau_{D}=-\frac{1}{\tau}=-\frac{\partial_{u} a}{\partial_{u} a_{D}} .
$$

Near the monopole point, $u=\Lambda^{2}$, the theory is in weak coupling regime, $\operatorname{Im} \tau_{D} \gg 1$, 
and the leading terms of of the expansions in $u-\Lambda^{2}$ are

$$
\begin{aligned}
& a_{D}=\frac{i}{2 \Lambda}\left(u-\Lambda^{2}\right)\left(1-\frac{u-\Lambda^{2}}{16 \Lambda^{2}}\right)\left\{1+\mathcal{O}\left[\left(\frac{u-\Lambda^{2}}{\Lambda^{2}}\right)^{2}\right]\right\} \\
& a=\frac{4 \Lambda}{\pi}+\frac{u-\Lambda^{2}}{2 \pi \Lambda}\left(1-\frac{u-\Lambda^{2}}{16 \Lambda^{2}}\right)\left(\ln \frac{32 \Lambda^{2}}{u-\Lambda^{2}}+1+\frac{u-\Lambda^{2}}{32 \Lambda^{2}}\right)\left\{1+\mathcal{O}\left[\left(\frac{u-\Lambda^{2}}{\Lambda^{2}}\right)^{2}\right]\right\} \\
& \tau_{D}=\frac{i}{\pi}\left(\ln \frac{32 \Lambda^{2}}{u-\Lambda^{2}}+\frac{u-\Lambda^{2}}{4 \Lambda^{2}}\right)\left\{1+\mathcal{O}\left[\left(\frac{u-\Lambda^{2}}{\Lambda^{2}}\right)^{2}\right]\right\} \\
& =\frac{i}{\pi}\left(\ln \frac{16 i \Lambda}{a_{D}}-\frac{3 i}{8} \frac{a_{D}}{\Lambda}\right)\left\{1+\mathcal{O}\left(\frac{a_{D}^{2}}{\Lambda^{2}}\right)\right\}
\end{aligned}
$$

The logarithmic singularity at $u=\Lambda^{2}$ (i.e. at $a_{D}=0$ ) is interpreted as the vacuum polarization of the monopoles whose mass $m_{M}=\sqrt{2}\left|a_{D}\right|$ vanishes at this point.

The logarithmic singularity is due to large distances of order $1 / m_{M} \gg 1 / \Lambda$, it makes clear that the Lagrangian (11) is not Wilsonean. To make description local one needs to "integrate in" light monopoles what leads to

$$
\begin{aligned}
\mathcal{L}_{\mathrm{Wils}}= & \frac{1}{8 \pi} \operatorname{Im} \int \mathrm{d}^{2} \theta \tilde{\tau}_{D}\left(A_{D}\right) W_{D}^{2}+\frac{1}{4 \pi} \operatorname{Im} \int \mathrm{d}^{2} \theta \mathrm{d}^{2} \bar{\theta} \tilde{\tau}_{D}\left(A_{D}\right) \bar{A}_{D} A_{D} \\
& +\int \mathrm{d}^{2} \theta \mathrm{d}^{2} \bar{\theta}\left[\bar{Q} \mathrm{e}^{V_{D}} Q+\overline{\tilde{Q}} \mathrm{e}^{-V_{D}} \tilde{Q}\right]+2 \operatorname{Re} \int \mathrm{d}^{2} \theta \mathcal{W}
\end{aligned}
$$

Here the $\mathcal{N}=1$ chiral superfields $Q$ and $\tilde{Q}$ describe the monopole hypermultiplet. The third term in (4) is the kinetic term for these fields, it includes also the gauge interaction of the monopoles. The superpotential $\mathcal{W}$ is then fixed by $\mathcal{N}=2 \mathrm{SUSY}$

$$
\mathcal{W}=\sqrt{2} \tilde{Q} A_{D} Q
$$

It reproduces correctly the monopole mass $m_{M}=\sqrt{2}\left|a_{D}\right|$.

The Wilsonean Lagrangian (四) is also supplied with a normalization point $M_{\mathrm{PV}}$ which plays a role of the ultraviolet cut off for the $\underset{\mathcal{Q}}{Q}, \tilde{Q}$ loops. This can be realized by introducing the Pauli-Villars regulators to the $Q, \tilde{Q}$ fields with the mass $M_{\mathrm{PV}}$. Then

$$
\tilde{\tau}_{D}\left(a_{D}\right)=\tau_{D}\left(a_{D}\right)-\frac{i}{\pi} \ln \frac{i M_{\mathrm{PV}}}{a_{D} \sqrt{2}}=\frac{i}{\pi}\left(\ln \frac{8 \sqrt{2} \Lambda}{M_{\mathrm{PV}}}-\frac{3 i}{8} \frac{a_{D}}{\Lambda}\right)
$$

matches the expression (3) after adding up the one-loop contribution of $Q, \tilde{Q}$. The coupling $\tilde{\tau}_{D}$ is nonsingular at $a_{D}=0$, its value at $a_{D}=0$

$$
\tilde{\tau}_{D}\left(a_{D}=0\right)=\frac{i}{\pi} \ln \frac{8 \sqrt{2} \Lambda}{M_{\mathrm{PV}}} .
$$

provides a weak coupling, $\operatorname{Im} \tilde{\tau}_{D}(0) \gg 1$. 
Moreover, for our further considerations we can safely neglect by the "nonperturbative" $a_{D} / \Lambda$ term in the expansion of $\tilde{\tau}_{D}$ limiting ourselves by $\tilde{\tau}_{D}(0)$. If the linear in $a_{D} / \Lambda$ correction were in the kinetic term of monopole fields it would be important. The absence of such corrections is seen from their absence in the logarithmic part of $\tau\left(a_{D}\right)$ in Eq. (3).

Now let us break $\mathcal{N}=2$ down to $\mathcal{N}=1$ by adding mass term for the adjoint matter in the microscopic $\mathrm{SU}(2)$ Seiberg-Witten theory

$$
\mathcal{W}_{\text {mass }}=\mu \operatorname{Tr} \Phi^{2}
$$

We can treat this breaking perturbatively when $\mu \ll \Lambda$. Then in the effective theory near the monopole point the perturbation $\Delta \mathcal{W}\left(A_{D}\right)$ of the superpotential can be expanded in powers of $A_{D}$,

$$
\Delta \mathcal{W}\left(A_{D}\right)=\mu u\left(A_{D}\right)=\mu \Lambda^{2}+\frac{i}{\sqrt{2}} \eta A_{D}+\frac{\mu_{D}}{2} A_{D}^{2}+\mathcal{O}\left(\mu A_{D}^{3} / \Lambda\right) .
$$

Here $u\left(a_{D}\right)$ is the inverse to $a_{D}(u)$ function, and the expansion (3) for $a_{D}(u)$ leads to

$$
\eta=-2 \sqrt{2} \mu \Lambda, \quad \mu_{D}=-\frac{1}{4} \mu
$$

Note that $\mu$ and $\Lambda$ are complex quantities, their phases can be changed by global $U(1)$ rotations. In considerations below we will often use this freedom to fix parameters $\eta$ and $\mu_{D}$ to be real and positive.

Minimizing the superpotential given by the sum of expressions (5) and (9) with respect to $Q, \tilde{Q}$ and $A_{D}$ we find that the Coulomb branch shrinks to the point [3]

$$
\left\langle a_{D}\right\rangle=0
$$

while the monopole condensate appears

$$
\langle\tilde{q} q\rangle=-\frac{i}{2} \eta
$$

Here $q, \tilde{q}$ are the scalar components of $Q, \tilde{Q}$. The monopole condensation breaks the $\mathrm{U}(1)$ gauge group and leads to confinement of electric charges.

Parameters $\eta$ and $\mu$ in the superpotential (9) play quite different role in the effective QED description of the theory. It was noted in [9] that the linear term in (9) is a generalization of the Fayet-Iliopoulos (FI) $D$-term. Moreover, at $\mu_{D}=0$ the $\eta$ term does not break $\mathcal{N}=2$ supersymmetry [9]. We confirm this by explicit calculations in the next section.

Breaking of $\mathcal{N}=2$ is due to the $\mu_{D}$ term in (9). As we will show it is this term which shifts the mass of the field $A_{D}$ away from the photon mass. Below considering the effective QED on general ground we view three parameters $\eta, \mu_{D}$ and $\tilde{\tau}(0)$ as independent ones. Within the Seiberg-Witten solution $\mu_{D}^{2} / \eta \sim \mu / \Lambda \ll 1$ in weak coupling. 


\section{Fayet-Iliopoulos term in $\mathcal{N}=2$ QED}

In this section we consider the $\mathcal{N}=2$ QED theory given by Eq. (4) and perturbed by the superpotential (9) with $\mu_{D}=0$ and nonvanishing $\eta$. As it was mentioned above we neglect by "nonperturbative" corrections $\left(a_{D} / \Lambda\right)^{k}$ and choose for simplicity $\tilde{\tau}_{D}(0)$ to be pure imaginary (i.e. taking the effective $\theta$ angle to be zero),

$$
\tilde{\tau}_{D}(0)=\frac{4 \pi i}{g^{2}}
$$

The theory becomes the $\mathcal{N}=2$ QED where the $\eta$ perturbation appears as the $\mathcal{N}=2$ generalization of the Fayet-Iliopoulos term. We show that although the $\eta$ perturbation does break the $\mathrm{SU}(2)_{R}$ global symmetry associated with $\mathcal{N}=2$ it does not break $\mathcal{N}=2$ supersymmetry. A remarkable feature of this theory is that all particles enter one long supermultiplet of $\mathcal{N}=2$. It means that there are no nontrivial central charges in this case in contrast with the Coulomb branch at $\eta=0$.

Having in mind vortex solutions we study then the reduction to $2+1$ dimensions. The reduced QED has $\mathcal{N}=4$ supersymmetry and we consider its superalgebra with central charges. In the $2+1$ dimensions strings are particle-like solutions with nonvanishing central charges. Indeed, as we will see in Sec. 4, the ANO string is BPS saturated. It is in agreement with Ref. [9].

\section{1 $\mathcal{N}=2$ superalgebra in $3+1$ dimensions}

Let us start rewriting the $\mathcal{N}=2 \mathrm{QED}$ in the component form. We will omit below index $D$ referring to the dual variables such as $A_{D}, V_{D}, W_{D}$ and will use the terminology of the electric description. The Lagrangian in component form then is

$$
\begin{aligned}
& \mathcal{L}_{\mathrm{QED}}=-\frac{1}{4 g^{2}} F_{\mu \nu}^{2}+\frac{1}{g^{2}}\left|\partial_{\mu} a\right|^{2}+\mathcal{D}_{\mu} \bar{\phi}_{f} \mathcal{D}^{\mu} \phi^{f}-U\left(\phi^{f}, a\right) \\
& +\frac{1}{g^{2}} \lambda^{f} i \sigma^{\mu} \partial_{\mu} \bar{\lambda}_{f}+\psi i \sigma^{\mu} \mathcal{D}_{\mu} \bar{\psi}+\tilde{\psi} i \sigma^{\mu} \mathcal{D}_{\mu} \overline{\tilde{\psi}}+\sqrt{2}\left[i \psi \lambda^{f} \bar{\phi}_{f}-\tilde{\psi} \lambda^{f} \phi_{f}-\psi \tilde{\psi} a+\text { h.c. }\right]
\end{aligned}
$$

where we introduce $\mathrm{SU}(2)_{R}$ doublet $\phi^{f}$ for scalar fields of the same electric (magnetic in the original dual description above) charge +1 ,

$$
\phi^{f}=\left(\begin{array}{c}
q \\
-i \overline{\tilde{q}}
\end{array}\right),
$$

(in our notation the bar is an equivalent of complex conjugation), and the covariant derivative $\mathcal{D}_{\mu}=\partial_{\mu}-i n_{e} A_{\mu}\left(n_{e}\right.$ is the electric charge). In the fermion sector $\lambda_{\alpha}^{f}$ is the $\mathrm{SU}(2)_{R}$ doublet of fermion fields in the gauge supermultiplet (gluino and the fermionic partner of $a$ ), while $\psi_{\alpha}$ and $\tilde{\psi}_{\alpha}$ are fermions from $Q$ and $\tilde{Q}$ superfields. Note, that without $\Delta \mathcal{W}$ of Eq. (9) the full flavor symmetry of the $\mathcal{N}=2$ QED is $\mathrm{SU}(2)_{R} \times \mathrm{U}(1)_{\bar{R}}$. The $\mathrm{U}(1)_{\bar{R}}$ charges of the fields are given in the Table 1 . 


\begin{tabular}{|c|c|c|c|c|c|c|}
\hline Fields & $a$ & $\phi^{f}$ & $A_{\mu}$ & $\lambda^{f}$ & $\psi$ & $\tilde{\psi}$ \\
\hline $\mathrm{U}_{\bar{R}}(1)$ charges & -1 & 0 & 0 & $-\frac{1}{2}$ & $\frac{1}{2}$ & $\frac{1}{2}$ \\
\hline
\end{tabular}

Table 1: $\mathrm{U}(1)_{\bar{R}}$ charges

The scalar potential $U(\phi, a)$ in Eq. (14) has the form

$$
U(\phi, a)=g^{2} \operatorname{Tr}\left[\bar{\phi}_{g} \phi^{f}-\frac{1}{2} \delta_{g}^{f}(\bar{\phi} \phi)-\frac{1}{2} \xi_{g}^{f}\right]^{2}+2|a|^{2} \bar{\phi}_{f} \phi^{f}
$$

where $\xi_{2}^{1}=\left(\xi_{1}^{2}\right)^{*}=\eta$ while diagonal components of $\xi_{g}^{f}$ are zero. Adding up these components generalizes $\xi_{g}^{f}$ to the traceless hermitian matrix equivalent to the triplet of $\mathrm{SU}_{R}(2)$,

$$
\xi_{g}^{f}=\xi_{a}\left(\tau_{a}\right)_{g}^{f}, \quad \xi_{1}=\operatorname{Re} \eta, \quad \xi_{2}=-\operatorname{Im} \eta
$$

The additional components $\xi_{3}=\xi_{1}^{1}=-\xi_{2}^{2}$ produce in the potential $U$ the extra piece

$$
U_{\mathrm{FI}}=g^{2}\left[-\xi_{3}\left(|q|^{2}-|\tilde{q}|^{2}\right)+\frac{1}{2}\left(\xi_{3}\right)^{2}\right] .
$$

It is easy to recognize $U_{\mathrm{FI}}$ as a component form of the standard Fayet-Iliopoulos (FI) term [12] in $\mathrm{U}(1)$ theory where in the superfield notation it is $\xi_{3} \int \mathrm{d}^{4} \theta V$. We see that the $\mathrm{SU}_{R}(2)$ symmetry unifies in the potential $U$ contributions from $F$-terms (due to linear in $A_{D}$ part of the superpotential $\Delta \mathcal{W}$ ) with the FI $D$-terms. For this reason we view pieces in $U$ associated with $\xi_{a}$ as generalized FI terms.

But this unification comes at a price: the nonvanishing $\xi$ explicitly breaks the $\mathrm{SU}(2)_{R}$ invariance as we see from Eq. (15). At first glance it suggests also breaking of the extended $\mathcal{N}=2$ supersymmetry. However, as it was noted in Ref. [9] within the brane construction, the extended $\mathcal{N}=2$ supersymmetry is not broken by the FI terms. Within the effective field theory (13) we are able to verify this explicitly by invariance under the following set of $\mathcal{N}=2$ transformations:

$$
\begin{aligned}
& \delta A_{\alpha \dot{\alpha}}=-2 i\left(\varepsilon_{\alpha}^{f} \bar{\lambda}_{\dot{\alpha} f}+\bar{\varepsilon}_{\dot{\alpha} f} \lambda_{\alpha}^{f}\right), \\
& \delta \lambda_{\alpha}^{f}=-\varepsilon^{\beta f} F_{\alpha \beta}+i \varepsilon_{\alpha}^{g} D_{g}^{f}+i \sqrt{2} \bar{\varepsilon}^{\dot{\alpha} f} \partial_{\alpha \dot{\alpha}} a, \\
& \delta a=-\sqrt{2} \varepsilon^{\alpha f} \lambda_{f \alpha}, \\
& \delta \phi^{f}=\sqrt{2} \varepsilon^{\alpha f} \psi_{\alpha}+i \sqrt{2} \bar{\varepsilon}_{\dot{\alpha}}^{f} \overline{\psi^{\dot{\alpha}}} \\
& \delta \psi_{\alpha}=-i \sqrt{2} \bar{\varepsilon}_{f}^{\dot{\alpha}} \mathcal{D}_{\alpha \dot{\alpha}} \phi^{f}+2 i \varepsilon_{\alpha}^{f} \phi_{f} \bar{a}, \\
& \delta \tilde{\psi}_{\alpha}=-\sqrt{2} \bar{\varepsilon}_{f}^{\dot{\alpha}} \mathcal{D}_{\alpha \dot{\alpha}} \bar{\phi}^{f}-2 \varepsilon_{\alpha}^{f} \bar{\phi}_{f} \bar{a} .
\end{aligned}
$$

Here $\varepsilon_{\alpha}^{f}, \bar{\varepsilon}_{g}^{\dot{\alpha}}$ are eight parameters of $\mathcal{N}=2$ transformations, and we use spinor notations for the Lorentz vectors, $X_{\alpha \dot{\alpha}}=\left(\sigma^{\mu}\right)_{\alpha \dot{\alpha}} X_{\mu}$. The generalized $D$-terms $D_{g}^{f}$ are

$$
D_{g}^{f}=-g^{2}\left[2 \bar{\phi}_{g} \phi^{f}-\delta_{g}^{f}(\bar{\phi} \phi)-\xi_{g}^{f}\right] .
$$


Technically, the preservation of $\mathcal{N}=2$ can be explained by noting that the SUSY transformations of generalized $D$-terms (fixed by the transformations of $\phi^{f}, \bar{\phi}_{g}$ ) do not depend on $\xi_{g}^{f}$ at all, i.e. the symmetry looks the same as at $\xi_{g}^{f}=0$.

Note that although the $\mathrm{SU}(2)_{R}$ symmetry is broken by nonvanishing $\xi_{g}^{f}$ the $\mathrm{U}(1)_{\bar{R}}$ is preserved at $\mu_{D}=0$. As an explanation we suggest that this $\mathrm{U}(1)_{\bar{R}}$ can be related to the $R$ symmetry of the microscopic theory which survives breaking by $\mu \operatorname{Tr} \Phi^{2}$ and broken only by quantum anomalies. As we will see in Sec. 5 in the effective QED theory $\mu_{D} \neq 0$ breaks $\mathrm{U}(1)_{\bar{R}}$ reflecting the effect of anomalies.

The most general $\mathcal{N}=2$ superalgebra preserving the Lorentz invariance in $3+1$ dimensions has the form:

$$
\left\{Q_{\alpha}^{f}, \bar{Q}_{\dot{\alpha} g}\right\}=2 \delta_{g}^{f} \sigma_{\alpha \dot{\alpha}}^{\mu} P_{\mu}, \quad\left\{Q_{\alpha}^{f}, Q_{\beta}^{g}\right\}=2 \epsilon^{f g} \epsilon_{\alpha \beta} \mathcal{Z}, \quad\left\{\bar{Q}_{\dot{\alpha}}^{f}, \bar{Q}_{\dot{\beta}}^{g}\right\}=2 \epsilon^{f g} \epsilon_{\dot{\alpha} \dot{\beta}} \overline{\mathcal{Z}},
$$

where $Q_{\alpha}^{f}, \bar{Q}_{\dot{\alpha} g}$ are supercharges $\left(\alpha, \dot{\alpha}=1,2\right.$ are Lorentz indices, $f, g=1,2$ are $\mathrm{SU}(2)_{R}$ indices), $P_{\mu}$ are the energy-momentum operators, $\mathcal{Z}$ is the complex central charge. It is well known that in gauge theories the real and imaginary parts of $\mathcal{Z}$ are related to electric and magnetic charges, and $|\mathcal{Z}|$ gives masses of charged BPS particles on the Coulomb branch of the Seiberg-Witten theory. In the $\mathcal{N}=2$ QED it is particularly simple: magnetic charges are absent and $\mathcal{Z}$ is given by $\int \mathrm{d}^{3} S^{\nu} \partial^{\mu}\left(a F_{\mu \nu}\right)$ which reduces to the electric charge times $\langle a\rangle$. Here $S^{\nu}$ is the time-like three-dimensional hyperplane.

Few comments on representations of the algebra. As we mentioned above the central charge in Eq. (20) gives masses of shortened BPS hypermultiplets to be proportional to their electric charges. It happens in the Coulomb phase of the $\mathcal{N}=2$ theory at $\xi=0$. Once we switch on a nonzero $\xi$ the charged matter fields $\phi^{f}$ develop vevs, see Eq. (11), and the theory is in the Higgs phase. Then electric charges are screened and the central charge vanishes. Therefore, there are no BPS particles at nonzero $\xi$, i.e. short supermultiplets are absent. Particularly, photon enters the long $\mathcal{N}=2$ multiplet containing eight bosonic states. Explicit calculations of the next subsection confirms this.

This consideration refers to particle-like objects in $3+1$ and does not include extended objects like ANO strings. To consider string solutions which break the $3+1$ Lorentz invariance it is convenient to view them as particle-like solitons in the world dimensionally reduced to $2+1$. We will do this in Sec. 3.3 .

\subsection{Mass spectrum}

In this subsection we explicitly work out the mass spectrum in the QED theory (13) to show that all particle states enter one long $\mathcal{N}=2$ supermultiplet. Consider scalar potential (15). By $\mathrm{SU}(2)_{R}$ rotation we can always put it in the form where only $\xi_{3}$ component is nonvanishing and positive,

$$
U(q, a)=\frac{g^{2}}{2}\left(|q|^{2}-|\tilde{q}|^{2}-\xi\right)^{2}+2 g^{2}|\tilde{q} q|^{2}+2|a|^{2}\left(|q|^{2}+|\tilde{q}|^{2}\right)
$$


where

$$
\xi=\sqrt{\xi_{a}^{2}}>0
$$

The $\xi$ dependent part of $U$ is nothing but mass terms for scalar fields $q$ and $\tilde{q}$ with $m_{q}^{2}=-g^{2} \xi$ and $m_{\tilde{q}}^{2}=g^{2} \xi$. At first glance giving masses to scalars but not to their fermionic partners does not preserve any supersymmetry at all. However, the negative

sign of $m_{q}^{2}$ signals instability of $q=0$ point. Supersymmetry is restored at the point of stability.

Indeed, the potential (21) is the sum of the positive-definite terms, and at the point where $U=0$ all $F$ and $D$ terms vanish. This happens at the following vevs:

$$
\langle q\rangle=\sqrt{\xi}, \quad\langle\tilde{q}\rangle=0, \quad\langle a\rangle=0 .
$$

We choose the field $q$ which develops vev to be real, it is just the unitary gauge for the vector field. Nonvanishing $\langle q\rangle$ breaks $\mathrm{U}(1)$ gauge group giving mass to the photon (see Eq. (13) ),

$$
m_{\gamma}^{2}=2 g^{2} \xi
$$

From the first term in the potential (21) we read off that the mass of the real scalar $q$ is the same as the photon mass,

$$
m_{q}^{2}=m_{\gamma}^{2}
$$

Together the real $q$ field and three polarization of the massive photon form up the boson part of the $\mathcal{N}=1$ massive vector multiplet. To extent that $\mathcal{N}=1$ is unbroken one scalar always has the same mass as the photon, no matter whether the $\mathcal{N}=2$ SUSY is broken or not. Of course, there are also four fermionic states in this multiplet.

What remains to calculate is masses of two complex field $\tilde{q}$ and $a$ (four bosonic states). In the quadratic expansion of $U$ these fields do not mix with others and their masses are also equal to that of photon,

$$
m_{\tilde{q}}^{2}=m_{a}^{2}=m_{\gamma}^{2} .
$$

Looking at the fermion part of the Lagrangian (14) it is simple to verify that all fermion masses are also equal to $m_{\gamma}$.

We see that all particles ( 8 boson plus 8 fermion states) have the same mass $m_{\gamma}$. They form the long $\mathcal{N}=2$ multiplet. It is what we anticipate based on on general consideration of $\mathcal{N}=2$ algebra.

\section{$3.3 \mathcal{N}=4$ superalgebra in $2+1$ dimensions}

Under dimensional reduction the $\mathcal{N}=2$ superalgebra of eight supercharges $Q_{\alpha}^{f}$, $\bar{Q}_{\dot{\alpha} g}$ in $3+1$ dimensions becomes the $\mathcal{N}=4$ superalgebra in $2+1$ dimensions. Our presentation here follows Ref. [14]. 
The global symmetry group in $2+1$ is $\mathrm{SL}(1,1 ; \mathbf{R}) \times \mathrm{SU}(2)_{R} \times \mathrm{SU}(2)_{\bar{R}}$. The first factor here is the Lorentz group in $2+1$. Spinors of this group are real, so the difference between dotted and undotted indices disappears in $2+1$. It results in the extra global symmetry: the flavor $\mathrm{U}(1)_{\bar{R}}$ we have in $3+1 \mathrm{D}$ becomes $\mathrm{SU}(2)_{\bar{R}}$ in $2+1 \mathrm{D}$.

Fields of QED given by the dimensional reduction of the action (13) to $2+1$ form the following multiplets of the global symmetry $\mathrm{SU}(2)_{R} \times \mathrm{SU}(2)_{\bar{R}}$. Bosonic fields of the $\mathcal{N}=4$ gauge supermultiplet are vector potentials $A_{\mu}(\mu=0,1,2)$, which are singlets, and the $\mathrm{SU}(2)_{\bar{R}}$ triplet of scalars $a_{\bar{g}}^{\bar{f}}$. We use the matrix notation,

$$
a_{\bar{g}}^{\bar{f}}=\left(\begin{array}{cc}
A_{3} & -\bar{a} \sqrt{2} \\
-a \sqrt{2} & -A_{3}
\end{array}\right) .
$$

for these scalars in the $\{0,1\}$ representation. The third scalar appeared in the reduction to $2+1$ from the spatial component $A_{3}$ of the gauge field.

Fermion fields $\lambda_{\alpha}^{f}, \bar{\lambda}_{\alpha}^{f}$ of the gauge supermultiplet form the $\{1 / 2,1 / 2\}$ representation of $\mathrm{SU}(2)_{R} \times \mathrm{SU}(2)_{\bar{R}}$. We denote the quadruplet of these fields as

$$
\lambda_{\alpha \bar{f}}^{f}=\left\{\lambda_{\alpha}^{f}, \bar{\lambda}_{\alpha}^{f}\right\}, \quad f, \bar{f}=1,2 .
$$

This representation can also be viewed as a vector $\lambda_{\alpha}^{\mu},(\mu=1,2,3,4)$ of $\mathrm{O}(4)$ group by the relation

$$
\lambda_{\alpha \bar{f}}^{f}=\lambda_{\alpha}^{\mu}\left(\tau^{\mu}\right)_{\bar{f}}^{f}, \quad \tau^{\mu}=(i, \vec{\tau})
$$

with real fields $\lambda_{\alpha}^{\mu}$ (which are just real and imaginary parts of the original complex ones). In the basis (28) the reality condition has the form

$$
\left(\lambda_{\alpha \bar{f}}^{f}\right)^{\dagger}=\lambda_{\alpha f}^{\bar{f}} \equiv \epsilon_{f f^{\prime}} \epsilon^{\bar{f} \bar{f}^{\prime}} \lambda_{\alpha \bar{f}^{\prime}}^{f^{\prime}}
$$

The matter hypermultiplet with the electric charge +1 contains scalars which form the $\{1 / 2,0\}$ representation - the doublet $\phi^{f}$, and fermions in $\{0,1 / 2\}$ representation the $\mathrm{SU}(2)_{\bar{R}}$ doublet $\psi^{\bar{f}}$,

$$
\psi^{\bar{f}}=\left(\begin{array}{c}
\psi \\
-i \overline{\tilde{\psi}}
\end{array}\right) .
$$

The appearance of the latter doublet is the only effect of the dimensional reduction for the hypermultiplet.

In terms of fields introduced above the $\mathcal{N}=4$ QED Lagrangian in $2+1$ takes the form

$$
\begin{aligned}
\mathcal{L}_{\mathrm{QED}}^{2+1}= & \frac{1}{4 g^{2}}\left[-F_{\mu \nu}^{2}+\partial_{\mu} a_{\bar{g}}^{\bar{f}} \partial^{\mu} a_{\bar{f}}^{\bar{g}}+2 i \bar{\lambda}_{\bar{f}}^{f} \gamma^{\mu} \partial_{\mu} \lambda_{f}^{\bar{f}}\right]+\mathcal{D}_{\mu} \bar{\phi}_{f} \mathcal{D}^{\mu} \phi^{f}+\bar{\psi}_{\bar{f}} \gamma^{\mu} \mathcal{D}_{\mu} \psi^{\bar{f}} \\
& -g^{2} \operatorname{Tr}\left(\bar{\phi}_{g} \phi^{f}-\frac{1}{2} \delta_{g}^{f} \bar{\phi} \phi-\frac{1}{2} \xi_{g}^{f}\right)^{2}-\frac{1}{2} \bar{\phi} \phi \operatorname{Tr}\left(a_{\bar{g}}^{\bar{f}}\right)^{2} \\
& -\bar{\psi}_{\bar{f}} \psi^{\bar{g}} a_{\bar{g}}^{\bar{f}}+\sqrt{2}\left[\psi^{\bar{f}} \gamma^{0} \lambda_{\bar{f}}^{f} \bar{\phi}_{f}+\text { h.c. }\right]
\end{aligned}
$$


where $\bar{\phi} \phi=\bar{\phi}_{f} \phi^{f}$ and we use the Majorana basis for the $2 \times 2$ matrices $\gamma^{\mu}$ :

$$
\gamma^{0}=\sigma_{2}, \quad \gamma^{1}=i \sigma_{3}, \quad \gamma^{2}=i \sigma_{1}
$$

Notice that for fermionic fields $\bar{\psi} \equiv \psi^{\dagger} \gamma^{0}$, i.e. the bar denotes the Dirac conjugation. Notice also that to have the Majorana basis after dimensional reduction of the third spatial direction we do the spinor rotation

$$
\psi^{\text {Majorana }}=\frac{1+i \sigma_{1}}{\sqrt{2}} \psi .
$$

for all fermion fields, namely these rotated fields are implied starting from Eq. (32).

The supertransformations (18) after the reduction to $2+1$ can be written as

$$
\begin{aligned}
& \delta A_{\mu}\left(\gamma^{\mu}\right)_{\alpha \beta}=-\left(\varepsilon_{\bar{f}}^{f}\right)_{\alpha}\left(\bar{\lambda}_{f}^{\bar{f}}\right)_{\beta}-\left(\bar{\varepsilon}_{\bar{f}}^{f}\right)_{\beta}\left(\lambda_{f}^{\bar{f}}\right)_{\alpha}, \\
& \delta a_{\bar{g}}^{\bar{f}}=-2 \bar{\varepsilon}_{f}^{\bar{f}} \lambda_{\bar{g}}^{f}+\delta_{\bar{g}}^{\bar{f}} \bar{\varepsilon}_{f}^{\bar{h}} \lambda_{\bar{h}}^{f}, \\
& \delta \lambda_{\bar{f}}^{f}=\frac{i}{2} F_{\mu \nu} \gamma^{\mu} \gamma^{\nu} \varepsilon_{\bar{f}}^{f}+D_{g}^{f} \varepsilon_{\bar{f}}^{g}-i \partial_{\mu} a_{\bar{f}}^{\bar{g}} \gamma^{\mu} \varepsilon_{\bar{g}}^{f}, \\
& \delta \phi^{f}=-\sqrt{2} \bar{\varepsilon}_{\bar{f}}^{f} \psi^{\bar{f}} \\
& \delta \psi^{\bar{f}}=-i \sqrt{2} \mathcal{D}_{\mu} \phi^{f} \gamma^{\mu} \varepsilon_{f}^{\bar{f}}-2 \sqrt{2} \phi^{f} a_{\bar{g}}^{\bar{f}} \varepsilon_{f}^{\bar{g}},
\end{aligned}
$$

where $\left(\varepsilon_{\bar{f}}^{f}\right)_{\alpha}=\left\{i \varepsilon_{\alpha}^{f}, i \bar{\varepsilon}_{\alpha}^{f}\right\}$ are eight Grassmann parameters satisfying to the reality condition similar to Eq. (30).

The transformations (35) imply that doublets of supercharges $Q_{\alpha}^{f}$ and $\bar{Q}_{\alpha}^{f} \equiv \epsilon^{f f^{\prime}}\left(Q_{\alpha}^{f^{\prime}}\right)^{\dagger}$ are combined into the quadruplet

$$
Q_{\alpha \bar{f}}^{f}=\left\{i \bar{Q}_{\alpha}^{f},-i Q_{\alpha}^{f}\right\}, \quad \bar{f}=1,2,
$$

which forms one irreducible representation $\{1 / 2,1 / 2\}$ of $\mathrm{SU}(2)_{R} \times \mathrm{SU}(2)_{\bar{R}}$, the same one we have introduced above for $\lambda_{\alpha \bar{f}}^{f}$.

The transformations (35) allow to determine the $\mathcal{N}=4$ superalgebra spanned by eight supercharges $Q_{\alpha}^{f \bar{f}}$. The most general form of the $\mathcal{N}=4$ superalgebra in $2+1$ is

$$
\left\{\left(Q_{\bar{f}}^{f}\right)_{\alpha},\left(\bar{Q}_{g}^{\bar{g}}\right)_{\beta}\right\}=2 \delta_{g}^{f} \delta_{\bar{g}}^{\bar{f}}\left(\gamma^{\mu}\right)_{\alpha \beta} P_{\mu}-2 \delta_{\alpha \beta}\left[\delta_{\bar{g}}^{\bar{f}} \mathcal{Z}_{g}^{f}+\delta_{g}^{f} \tilde{\mathcal{Z}}_{\bar{g}}^{\bar{f}}\right]
$$

where the traceless $\mathcal{Z}_{g}^{f}, \tilde{\mathcal{Z}}_{2 \bar{g}}^{\bar{f}}$ are six central charges. The QED with generalized FI terms given by Eq. (32) leads to the following expression for these central charges,

$$
\mathcal{Z}_{g}^{f}=L \xi_{g}^{f} \int \mathrm{d}^{2} S^{\mu} F_{\mu}^{*}=2 \pi n L \xi_{g}^{f}, \quad \tilde{\mathcal{Z}}_{\bar{g}}^{\bar{f}}=0
$$

where $L$ is the total length of the reduced dimension, $\mathrm{d}^{2} S^{\mu}$ is the element of the timelike two-dimensional hyperplane, and $F_{\mu}^{*}=\epsilon_{\mu \nu \gamma} F^{\nu \gamma} / 2$. The integral $\int \mathrm{d}^{2} S^{\mu} F_{\mu}^{*}$ is the magnetic flux which is quantized, $\int \mathrm{d}^{2} S^{\mu} F_{\mu}^{*}=2 \pi n$. 
The $\mathcal{N}=4$ superalgebra (37) with the central charges $(38)$ generalizes the $\mathcal{N}=2$ algebra for the standard FI term [13, 15]. The generalization amounts to introduction of SU $(2)_{R}$ group and to substitution of the FI $D$-term coefficient $\xi_{3}$ by the matrix $\xi_{g}^{f}$. Nonvanishing $\xi_{g}^{f}$ explicitly breaks the $\mathrm{SU}(2)_{R}$ flavor symmetry, the $\mathrm{SU}(2)_{\bar{R}}$ is preserved, however, because $\tilde{\mathcal{Z}}_{\bar{g}}^{\bar{f}}=0$.

To consider representation of the algebra (37) let us write it down in the rest frame where $P_{\mu}=(M, 0,0)$ via four complex charges

$$
Q_{\bar{f}}^{f}=\frac{1}{\sqrt{2}}\left[\left(Q_{\bar{f}}^{f}\right)_{1}+i\left(Q_{\bar{f}}^{f}\right)_{2}\right]
$$

instead of eight real $Q_{\alpha}^{f \bar{f}}$. For these supercharges with no spinor index we find from Eq. (37)

$$
\left\{Q_{\bar{f}}^{f},\left(Q^{\dagger}\right)_{g}^{\bar{g}}\right\}=M \delta_{g}^{f} \delta_{\bar{g}}^{\bar{f}}-\delta_{\bar{g}}^{\bar{f}} \mathcal{Z}_{g}^{f}-\delta_{g}^{f} \tilde{\mathcal{Z}}_{\bar{g}}^{\bar{f}}, \quad\left\{Q_{\bar{f}}^{f}, Q_{\bar{g}}^{g}\right\}=0, \quad\left\{\left(Q^{\dagger}\right)_{f}^{\bar{f}},\left(Q^{\dagger}\right)_{g}^{\bar{g}}\right\}=0 .
$$

The positive definiteness of the first anticommutator implies the bound

$$
M \geq \operatorname{Max}\left\{\sqrt{\mathcal{Z}_{g}^{f} \mathcal{Z}_{f}^{g} / 2}, \sqrt{\tilde{\mathcal{Z}}_{\bar{g}}^{\bar{f}} \tilde{\mathcal{Z}}_{\bar{f}}^{\bar{g}} / 2}\right\}
$$

for the mass in $2+1 \mathrm{D}$. If the central charges $\mathcal{Z}$ and $\tilde{\mathcal{Z}}$ are both nonvanishing the BPS multiplet saturating the bound consists of four bosonic and four fermionic states and preserves $1 / 4$ of the $\mathcal{N}=4 \mathrm{SUSY}$. In our case when $\tilde{\mathcal{Z}}=0$ the bound $\sqrt{\mathcal{Z}_{g}^{f} \mathcal{Z}_{f}^{g} / 2}$ is saturated by BPS multiplet containing two bosonic plus two fermionic states, 1/2 of the $\mathcal{N}=4$ SUSY is preserved.

The mass of the string in $3+1$ dimensions is proportional to its length, $M=T L$, and Eq. (41) becomes the bound for the string tension $T$,

$$
T \geq 2 \pi|n| \xi
$$

We used here expressions (38) for the central charges and the definition $\xi=\sqrt{\xi_{g}^{f} \xi_{f}^{g} / 2}$ introduced earlier in Eq. (22). The BPS strings saturate this bound.

The BPS strings form the short (2 boson +2 fermion) supermultiplet of $\mathcal{N}=4$. From Eq. (40) with the central charges (38) one can see that the invariant subalgebra is formed by four supercharges

$$
\frac{1}{2}\left(\delta_{g}^{f}+\frac{1}{\xi} \xi_{g}^{f}\right) Q_{\bar{f}}^{g}, \quad \frac{1}{2}\left(\delta_{f}^{g}+\frac{1}{\xi} \xi_{f}^{g}\right)\left(Q^{\dagger}\right)_{g}^{\bar{f}} .
$$

These supercharges annihilate the BPS states, we will use this for construction of the BPS string solutions in the next section. 


\section{The BPS solution for string}

In this section we consider the ANO string in QED with FI terms. We generalize to the $\mathcal{N}=2$ supersymmetry (in $3+1 \mathrm{D}$ ) the results of Ref. [16] obtained in the $\mathcal{N}=1$ case (see also [15]).

Let us start from reminding basic facts about the ANO string in framework of the non-supersymmetric Abelian Higgs model. The model contains one complex scalar field $\varphi$ interacting with electromagnetic field,

$$
S_{\mathrm{AH}}=\int \mathrm{d}^{4} x\left\{-\frac{1}{4 g^{2}} F_{\mu \nu}^{2}+\left|\mathcal{D}_{\mu} \varphi\right|^{2}-\frac{\lambda}{2}\left(|\varphi|^{2}-\xi\right)^{2}\right\} .
$$

The field $\varphi$ develops vev, $\varphi^{2}=\xi$, the $\mathrm{U}(1)$ gauge group is broken. Photon acquires the mass, $m_{\gamma}^{2}=2 g^{2} \xi$, see Eq. (24), the Higgs mass is equal to $m_{H}^{2}=2 \lambda \xi$.

For an arbitrary $\lambda$ the Higgs mass differs from that of the photon. The ratio of the photon mass to the Higgs mass is an important parameter, in the theory of superconductivity it characterizes the type of superconductor, see e.g. Ref. [17. Namely, for $m_{H}<m_{\gamma}$ we have the type I superconductor, while for $m_{H}>m_{\gamma}$ we have the type II. This is related to the fact that scalar field produces an attraction for two vortices, while the electromagnetic field produces a repulsion.

The boundary separating superconductors of the I and II type corresponds to $m_{H}=m_{\gamma}$, i.e. to the special value of the quartic coupling $\lambda$,

$$
\lambda=g^{2} .
$$

In this case vortices do not interact. It is well known that vanishing of the interaction at $m_{H}=m_{\gamma}$ can be explained by the BPS nature of the ANO strings. The ANO string satisfy the first order equations and saturate the Bogomolny bound [18]. This bound follows from the following representation for the string tension $T$,

$$
T=2 \pi \xi n+\int \mathrm{d}^{2} x\left\{\frac{1}{2 g^{2}}\left[F_{12}+g^{2}\left(|\varphi|^{2}-\xi\right)\right]^{2}+\left|\left(\mathcal{D}_{1}+i \mathcal{D}_{2}\right) \varphi\right|^{2}\right\} .
$$

Here indices 1,2 denote coordinates transverse to the axis of the vortex. The minimal value of the tension is reached when both positive terms in the integrand of Eq. (46) vanish f,

$$
F_{12}+g^{2}\left(|\varphi|^{2}-\xi\right)=0, \quad\left(\mathcal{D}_{1}+i \mathcal{D}_{2}\right) \varphi=0 .
$$

The string tension becomes

$$
T=2 \pi \xi n,
$$

\footnotetext{
${ }^{2}$ In the context of the Landau-Ginzburg approach to superconductivity the same system of first order differential equations was derived by G. Sarma at early sixties in application to the case of vanishing surface energy, see Ref. [17].
} 
where the winding number $n$ counts the quantized magnetic flux $\int \mathrm{d}^{2} x F_{12}=2 \pi n$ (we assume that $n$ is positive). The linear dependence of string tensions on $n$ implies absence of their interaction.

For the $n=1$ vortex it is convenient to introduce the profile functions $s(r)$ and $f(r)$,

$$
\varphi(x)=\sqrt{\xi} s(r) \mathrm{e}^{i \alpha}, \quad A_{n}(x)=-\epsilon_{n m} \frac{x_{m}}{r^{2}} f(r),
$$

where $r=\sqrt{x_{1}^{2}+x_{2}^{2}}$ is the distance and $\alpha$ is the polar angle in the $(1,2)$ plane. Then from Eqs. (47) we get

$$
\frac{1}{\rho} \frac{\mathrm{d} f}{\mathrm{~d} \rho}+s^{2}-1=0, \quad \rho \frac{\mathrm{d} s}{\mathrm{~d} \rho}+(f-1) s=0 .
$$

Here $\rho=g \sqrt{\xi} r$ is dimensionless distance. The profile functions are real and satisfy the boundary conditions

$$
s(0)=f(0)=0, \quad s(\infty)=f(\infty)=1,
$$

which ensures that the scalar field reaches its vev (23) at the infinity and the vortex carries one unit of the magnetic flux. The equations (50) with the boundary conditions (51) lead to the unique solution for the profile functions (although an analytic form of the solution is not found).

Returning to the supersymmetric QED we see that the tension (48) coincides with the bound (41) derived from the $\mathcal{N}=4$ algebra. Moreover, vanishing of both terms in the integrand of Eq. (46) is in correspondence to annihilation of the bosonic solution by the action of four supercharges (43). Indeed, let us choose $\xi_{1,2}=0, \xi_{3}=\xi$. Then, the set (43) becomes $Q^{1 \bar{f}}, \bar{Q}_{1 \bar{f}}$. The action of these supercharges on bosonic fields can be read off supersymmetry transformations (35) for $\delta \lambda$ and $\delta \psi$ with following substitution of parameters

$$
\left(\varepsilon_{\bar{f}}^{f}\right)_{\alpha} \rightarrow-\frac{i}{\sqrt{2}}\left[\delta_{1}^{f}\left(\delta_{\alpha}^{1}-i \delta_{\alpha}^{2}\right) \bar{\varepsilon}_{\bar{f}}-\delta_{2}^{f}\left(\delta_{\alpha}^{1}+i \delta_{\alpha}^{2}\right) \varepsilon_{\bar{f}}\right]
$$

Equating $\delta \lambda$ and $\delta \psi$ for these transformations to zero we get

$$
\begin{aligned}
& F_{12}-D_{1}^{1}=0, \quad F_{01}=F_{02}=0, \quad D_{2}^{1}=D_{1}^{2}=0, \quad \partial_{1} a_{\bar{g}}^{\bar{f}}=\partial_{2} a_{\bar{g}}^{\bar{f}}=0, \\
& \left(\mathcal{D}_{1}+i \mathcal{D}_{2}\right) \phi^{1}=0, \quad\left(\mathcal{D}_{1}-i \mathcal{D}_{2}\right) \phi^{2}=0, \quad \phi^{f} a_{\bar{g}}^{\bar{f}}=0 .
\end{aligned}
$$

Here $D_{g}^{f}$ is defined by Eq. (19) and we imply that the bosonic configuration is time independent. With our choice of the $\xi$ orientation it follows from Eqs. (53) that $\phi^{2}=$ $a_{\bar{g}}^{\bar{f}}=0$. Remaining equations coincide with Eq. (47) for Abelian Higgs strings (up to correspondence $\phi^{1} \rightarrow \varphi$ ).

Let us discuss now the fermion zero modes of the $n=1$ vortex. We demonstrated above that the vortex configuration is annihilated by action of four (out of eight) supercharges. Action of remaining four supercharges on the vortex configuration generates 
four zero modes. We get these modes substituting $\varepsilon_{\bar{f}}^{f}$ as

$$
\left(\varepsilon_{\bar{f}}^{f}\right)_{\alpha} \rightarrow \frac{i}{\sqrt{2}}\left[\delta_{1}^{f}\left(\delta_{\alpha}^{1}+i \delta_{\alpha}^{2}\right) \bar{\varepsilon}_{\bar{f}}-\delta_{2}^{f}\left(\delta_{\alpha}^{1}-i \delta_{\alpha}^{2}\right) \varepsilon_{\bar{f}}\right]
$$

in the transformations (35):

$$
\begin{aligned}
\left(\lambda_{\bar{f}}^{f}\right)_{\alpha} & =i \sqrt{2} g^{2}\left(\xi-\varphi^{2}\right)\left\{\delta_{1}^{f} \varepsilon_{\bar{f}}\left(\begin{array}{l}
1 \\
i
\end{array}\right)+\delta_{2}^{f} \bar{\varepsilon}_{\bar{f}}\left(\begin{array}{c}
1 \\
-i
\end{array}\right)\right\} \\
& =i \sqrt{2} g^{2} \xi\left(1-s^{2}\right)\left\{\delta_{1}^{f} \varepsilon_{\bar{f}}\left(\begin{array}{l}
1 \\
i
\end{array}\right)+\delta_{2}^{f} \bar{\varepsilon}_{\bar{f}}\left(\begin{array}{c}
1 \\
-i
\end{array}\right)\right\} \\
\psi_{\alpha}^{\bar{f}} & =i \bar{\varepsilon}^{\bar{f}}\left(\mathcal{D}_{1}-i \mathcal{D}_{2}\right) \varphi\left(\begin{array}{l}
1 \\
i
\end{array}\right)=i 2 \sqrt{\xi} \bar{\varepsilon}^{\bar{f}} \frac{(1-f) s}{r}\left(\begin{array}{l}
1 \\
i
\end{array}\right) .
\end{aligned}
$$

Four fermion zero modes obtained above are in correspondence with shortening of the supermultiplet of vortices: two bosonic and two fermionic states. The shortening guarantees the BPS nature of the vortex and the exactness of the mass formula (48).

Our consideration above was done in the frame where the $\mathrm{SU}(2)_{R}$ vector $\xi_{a}$ was oriented along the third axis, $\xi_{1,2}=0$. In this frame $\xi$ has the meaning of the standard FI $D$-term. The case of FI $F$-terms when $\xi_{3}=0, \xi_{1,2} \neq 0$ can be obtained from the case of FI $D$-term by $\mathrm{SU}(2)_{R}$ rotation. In particular, for the real parameter $\eta$ we have $\xi_{1}=\eta$ as the only nonvanishing component (see Eq. (16)). Then the above mentioned rotation is the rotation around the second axis on the angle $\pi / 2$. For $\mathrm{SU}(2)_{R}$ doublet $\phi^{f}$ this yields

$$
\phi_{F}^{f}=U_{g}^{f} \phi_{D}^{g}, \quad U=\mathrm{e}^{-i\left(\tau_{2} / 2\right)(\pi / 2)}=\frac{1}{\sqrt{2}}\left(\begin{array}{rr}
1 & -1 \\
1 & 1
\end{array}\right),
$$

where indices $D$ and $F$ denote fields in the theories with FI $D$-term and $F$-term respectively. For the string solution it means that

$$
q_{F}=-i \overline{\tilde{q}}_{F}=\frac{\varphi}{\sqrt{2}}
$$

with other fields unchanged. The same $\mathrm{SU}(2)_{R}$ rotation should be applied to fermion zero modes,

$$
\lambda_{F \bar{f}}^{f}=U_{g}^{f} \lambda_{D \bar{f}}^{f} .
$$

\section{The mass term perturbation}

In this section in addition to the linear in $A_{D}$ term in $\Delta \mathcal{W}$, see Eq. (9), we also switch on the mass term for $A_{D}$ (the term $\mu_{D} A_{D}^{2} / 2$ in Eq. (9)) in our low energy QED (4). 
It shifts the mass of the fermion field in the $A_{D}$ supermultiplet, and also changes the scalar potential to the form

$$
U(q, a)=\frac{g^{2}}{2}\left(|q|^{2}-|\tilde{q}|^{2}\right)^{2}+2 g^{2}\left|\tilde{q} q+\frac{i}{2} \eta+\frac{\mu_{D}}{\sqrt{2}} a\right|^{2}+2|a|^{2}\left(|q|^{2}+|\tilde{q}|^{2}\right) .
$$

In the covariant form (15) an introduction of $\mu_{D}$ can be viewed as an addition to the constant parameters $\xi_{g}^{f}$ terms $\Delta \xi_{g}^{f}$ which are linear in the fields $a$ and $\bar{a}$,

$$
\Delta \xi_{2}^{1}=\left(\Delta \xi_{1}^{2}\right)^{*}=-i \sqrt{2} \mu_{D} a .
$$

The field $a$ has nonzero $\mathrm{U}(1)_{\bar{R}}$ charge, see Table 1 . In the $2+1 \mathrm{D}$ reduced theory $a$ is a component of the $\mathrm{SU}(2)_{\bar{R}}$ triplet. Thus, adding $\Delta \xi$ of the form (60) breaks the $\mathrm{U}(1)_{\bar{R}}$ global symmetry (as well as $\mathrm{SU}(2)_{\bar{R}}$ in the $2+1 \mathrm{D}$ reduction).

Moreover, as we already mentioned, the mass term for $A_{D}$ breaks $\mathcal{N}=2$ supersymmetry down to $\mathcal{N}=1$. We show this explicitly below calculating the masses of boson fields. Later on we study what happens to the ANO vortex at $\mu_{D} \neq 0$ and show that it becomes a non-BPS object.

Note, that in the Seiberg-Witten theory the $\mathrm{SU}(2)_{R}$ vector $\Delta \xi$ contains part along the vector $\xi$. This is a generic situation. In the special case of QED with the usual $D$ type FI term an introduction of $\mu_{D}$ produces $\Delta \xi$ which is orthogonal to $\xi$. In this case the vortex remains BPS-saturated although $\mathcal{N}=2$ SUSY is broken down to $\mathcal{N}=1$. The dynamical side of this property of QED with $D$-term is that the scalar field $q$ which gets vev is in fact the superpartner of the photon in the massive $\mathcal{N}=1$ multiplet, so their masses are equal.

\subsection{Mass spectrum}

The minimum of the potential (59) coincides with the one in obtained for $\mu_{D}=0$, i.e.

$$
\langle q\rangle=i\langle\tilde{q}\rangle=\sqrt{\frac{\eta}{2}}, \quad\langle a\rangle=0,
$$

where we for simplicity consider $\eta$ to be real and positive (it is always possible to do by $U(1)$ rotation). Expanding the fields $q, \tilde{q}$ and $a$ around their vevs in the potential (59) and extracting quadratic in fluctuations terms we get after some algebra the $6 \times 6$ mass matrix

$$
\mathcal{M}^{2}=m_{\gamma}^{2}\left(\begin{array}{cccccc}
1 & 0 & 0 & 0 & \omega / \sqrt{2} & 0 \\
0 & 1 & 0 & 0 & \omega / \sqrt{2} & 0 \\
0 & 0 & 1 / 2 & 1 / 2 & 0 & \omega / \sqrt{2} \\
0 & 0 & 1 / 2 & 1 / 2 & 0 & \omega / \sqrt{2} \\
\omega / \sqrt{2} & \omega / \sqrt{2} & 0 & 0 & 1+\omega^{2} & 0 \\
0 & 0 & \omega / \sqrt{2} & \omega / \sqrt{2} & 0 & 1+\omega^{2}
\end{array}\right)
$$


where $m_{\gamma}^{2}=2 g^{2} \eta$ and we introduce the dimensionless parameter

$$
\omega=\frac{g \mu_{D}}{\sqrt{2 \eta}}
$$

implying that $\eta$ and $\mu_{D}$ are real and positive.

Calculating the determinant of the matrix $\mathcal{M}^{2}-\lambda m_{\gamma}^{2} I$ we find the eigenvalues of the mass matrix. We have

$$
\operatorname{det}\left(\mathcal{M}^{2}-\lambda m_{\gamma}^{2} I\right)=-m_{\gamma}^{12} \lambda(1-\lambda)\left[(1-\lambda)^{2}-\lambda \omega^{2}\right]^{2} .
$$

In the limit of small $\mathcal{N}=2$ breaking, $\omega \ll 1$, we have for six eigenvalues

$$
m_{i}^{2}=m_{\gamma}^{2}(0,1,1-\omega, 1-\omega, 1+\omega, 1+\omega)
$$

We interpret this result as follows. The massless state is "eaten up" by the Higgs mechanism and the scalar with the mass $m_{\gamma}$ is the scalar superpartner of photon in the massive vector $\mathcal{N}=1$ supermultiplet (see Sec. 3). A new phenomenon occurs with other four real scalars. The $\mathcal{N}=2$ hypermultiplet is split into two $\mathcal{N}=1$ chiral fields (each contains two real scalars) with different masses $m_{\gamma}^{2}(1 \pm \omega)$. Thus, we see explicitly that $\mathcal{N}=2$ supersymmetry is broken.

Consider also the opposite limit of strong $\mathcal{N}=2$ breaking, $\omega \gg 1$. We will use this limit in the next section. In this limit the masses of a former $\mathcal{N}=2$ hypermultiplet are split as follows

$$
m_{3}=m_{4}=m_{\gamma} \omega=g^{2} \mu_{D}, \quad m_{5}=m_{6}=\frac{m_{\gamma}}{\omega}=2 \frac{\eta}{\mu_{D}} .
$$

The limit $\omega \gg 1$ can be understood as taking the FI parameter $\eta$ to zero. Then it is clear that the first set of masses, $m_{3,4}$, in Eq. (66) refers to the $\mathcal{N}=1$ chiral field $A$. Its mass approaches the value $g^{2} \mu_{D}$ (note the $1 / g^{2}$ normalization of the kinetic energy for $A$ ). Moreover, the $\mathrm{U}(1)$ gauge symmetry is restored in the limit $\eta=0$ and the four real scalars $(q, \tilde{q})$ become massless, $m_{1}=0, m_{2} \rightarrow 0, m_{5}=m_{6} \rightarrow 0$.

\subsection{The string: qualitative remarks.}

In this subsection we make a few qualitative remarks on the ANO vortex solution for the theory with non-zero $\mu_{D}$. First, let us note that the ansatz (57) with the field $a=0$ does not work any longer. To see this consider the classical equation of motion for $a$. We have

$$
-\left(\partial_{x}^{2}+\partial_{y}^{2}\right) a+\sqrt{2} g^{4} \bar{\mu}_{D}\left(\tilde{q} q+\frac{i}{2} \eta+\frac{\mu_{D}}{\sqrt{2}} a\right)+2 g^{2} a(\bar{q} q+\overline{\tilde{q}} \tilde{q})=0
$$

We are looking for the vortex solution for which monopole fields $q, \tilde{q}$ are not identically equal to their vevs and acquire a non-trivial coordinate dependence. Then we see that 
$a=0$ does not satisfy (67) at non-zero $\mu_{D}$. It is clear that now all three scalar fields $q, \tilde{q}$ and $a$ have a non-trivial coordinate dependence.

We see that now the problem does not reduce to the simple Abelian Higgs model of the type (44) with a single complex scalar for which vortex solutions are well understood. In principle, it is possible to develop a perturbation theory around the $\mu_{D}=0$ solution in powers of $\mu_{D}$. This is done in the recent paper [19. The result of numerical solution of equations of motion for vortex in [19] seems to be in qualitative agreement with our conclusion presented in the next section.

In the next section we take the following route. We find the explicit vortex solution for a particular range of parameters $\eta$ and $\mu_{D}$ using our freedom to vary them independently within our effective QED description. The string tension in this limit shows the type I superconductivity.

Now let us make a comment about fermion zero modes of the vortex. Since the $\mathcal{N}=2$ SUSY is broken down to $\mathcal{N}=1$ we have only four SUSY generators now. It is clear on the other hand that the number of fermion zero modes of the vortex cannot jump as we switch on parameter $\mu_{D}$. Fermion zero modes are given by small deformation of the ones in Eqs. (55), (58). Thus we have four fermion zero modes what corresponds to two fermion states in the supermultiplet of vortex states. It means that this supermultiplet is not a short one for $\mathcal{N}=1$ and the vortex is not BPS-saturated at non-zero $\mu_{D}$.

\section{Large $\mu_{D}$ limit}

In this section we use the possibility to consider $\eta$ and $\mu_{D}$ as an independent parameters ignoring the relations (10), of the Seiberg-Witten theory. Namely, we consider the limit of large $\mu_{D}, \mu_{D}^{2} \gg \eta$. Note, that we still keep $\eta \ll \Lambda^{2}$ and $\mu_{D} \ll \Lambda$ in order to use the weak coupling QED description.

If the field $A$ is heavy we can integrate it out. From (5) and (9) we get the effective superpotential depending on monopole fields $Q, \tilde{Q}$ only (see [7, 20] where the similar integration is done for $\eta=0$ )

$$
\mathcal{W}_{\mu_{D} \rightarrow \infty}=-\frac{i}{\mu_{D}}\left(\tilde{Q} Q+i \frac{\eta}{2}\right)^{2} .
$$

The scalar potential of the Abelian Higgs model which follows from this superpotential reads

$$
U(q, \tilde{q})_{\mu_{D} \rightarrow \infty}=\frac{g^{2}}{2}\left(|q|^{2}-|\tilde{q}|^{2}\right)+\frac{4}{\mu_{D}^{2}}\left(|q|^{2}+|\tilde{q}|^{2}\right)\left|\tilde{q} q+i \frac{\eta}{2}\right|^{2}
$$

The first term here comes from the $D$-components of gauge multiplet (see (59)), while the second one comes from the superpotential (68). 
The potential (69) has a minimum at $q=\tilde{q}=0$ with unbroken $U(1)$ gauge group as well as the one written down in (61) for $q$-fields with the broken gauge group. Below we concentrate on the latter one.

Calculating the $4 \times 4$ mass matrix near this vacuum we obtain one zero eigenvalue (corresponding to the state "eaten up" by the Higgs mechanism), another one equal to $m_{\gamma}$, Eq. (24), (the scalar superpartner of the photon), while other two masses are

$$
m_{H}=\frac{m_{\gamma}}{\omega}
$$

where $\omega$ is introduced in (63). This is the mass of two scalars in $\mathcal{N}=1$ chiral multiplet. Note, as an additional check, that the mass of a chiral field in (70) coincides with $m_{5,6}$ in Eq. (66), as it is expected.

In order to use the effective potential (69) we should consider $\mu_{D}$ large enough to ensure that the mass of the scalar $a$ is much larger than both photon mass $m_{\gamma}$ and the mass of chiral field (70). The mass of $a$ can be rewritten in terms of $\omega$ as

$$
m_{a}=g^{2} \mu_{D}=m_{\gamma} \omega .
$$

We see that (69) is valid if

$$
\omega \gg 1 \text {. }
$$

We impose this condition below in this section.

Now consider ANO vortex in the QED with the potential (69). By symmetry between $q$ and $\tilde{q}$ it is clear that the classical solution corresponds to the ansatz with a single complex scalar $\varphi$

$$
q=\frac{1}{\sqrt{2}} \varphi, \quad \tilde{q}=-\frac{i}{\sqrt{2}} \bar{\varphi} .
$$

Substituting (73) into (69) we arrive at the following Abelian Higgs model

$$
S=\int \mathrm{d}^{4} x\left\{\frac{1}{4 g^{2}} F_{\mu \nu}^{2}+\left|\mathcal{D}_{\mu} \varphi\right|^{2}+\frac{1}{\mu_{D}^{2}}|\varphi|^{2}\left(|\varphi|^{2}-\eta\right)^{2}\right\} .
$$

The mass of the scalar $\varphi$ near the vacuum $\langle\varphi\rangle=\sqrt{\eta}$ coincides with the $m_{H}$ in Eq. (70). At $\omega \gg 1$ we have

$$
m_{H} \ll m_{\gamma} .
$$

Although the Higgs potential in (74) is not a standard one (see (44)) the condition (75) suggests that the superconductivity in the model (74) is of type I. We now show that this is indeed the case.

The vortex solutions in the Abelian Higgs model with a standard Higgs potential (44) under condition (75) have been studied in [21]. To the leading order in $\log \left(m_{\gamma} / m_{H}\right)$ the vortex solution has the following structure: the electromagnetic field is confined in a core with the radius

$$
R_{g} \sim \frac{1}{m_{\gamma}} \log \frac{m_{\gamma}}{m_{H}}
$$


while the scalar field $\varphi$ is close to zero inside the core. Outside the core the electromagnetic field is very small. At intermediate distances

$$
R_{g} \ll r \ll \frac{1}{m_{H}}
$$

( $r$ is the distance from the center of vortex in (1,2) plane) the scalar field satisfies the free equation of motion. Its solution reads [21]

$$
s(r)=1-\frac{\log \left(r m_{H}\right)}{\log \left(R_{g} m_{H}\right)},
$$

where we assume the standard ansatz (49) for $\varphi$ and $A_{\mu}$. At large distances $r \gg 1 / m_{H}$ $\varphi$ approaches its vev as

$$
|\varphi|-\sqrt{\eta} \sim \sqrt{\eta}[s(r)-1] \sim \exp \left(-m_{H} r\right) .
$$

The main contribution to the string tension comes from the logarithmically large region (77), where scalar field is given by (78). The result for the string tension is [21]

$$
T=\frac{2 \pi \eta}{\log \left(m_{\gamma} / m_{H}\right)}
$$

It comes from the kinetic energy of the scalar field in Eq. (44) (the "surface" energy).

Now it is clear that the vortex solution in the model (74) to the leading order has the same structure once the condition (75) is imposed. To see this observe that the main contribution to the string tension comes from the region (77) where the scalar field is given by the solution (78) of free equations of motion. The details of the scalar potential are not essential in this region. They become important in the region $r \sim 1 / m_{H}$, but the "volume" energy coming from this region is suppressed by an extra powers of $\log \left(m_{\gamma} / m_{H}\right)$ as compared with the one in Eq. (80).

We conclude that at large $\omega \gg 1$ our effective QED behaves as a type I superconductor and the string tension of ANO vortex is given by

$$
T=\frac{2 \pi \eta}{\log \omega} .
$$

Now let us discuss what does this conclusion means for the Seiberg-Witten theory. We have to reduce $\mu_{D}$ going to the region $\mu_{D}^{2} \ll \eta$ where the relations (10) are fulfilled. It is clear that while we continuously reduce $\mu_{D}$ the string tension $T$ stays below the BPS value $2 \pi \eta$, ensuring the type I superconductivity. The reason for this is that the number of states in the string multiplet cannot jump. As we discuss above for $\mu_{D}^{2} \gg \eta$ the string is not a BPS state and belongs to the "long" supermultiplet. Suppose that as we reduce $\mu_{D}$ the string tension crosses the bound $2 \pi \eta$ at some finite $\mu_{D}$. This would mean that the string becomes BPS-saturated and should belong to the "short" multiplet. This hardly can happen. Thus we conclude that

$$
T<2 \pi \eta
$$


for any nonzero $\mu_{D}$. The result (82) ensures the type I superconductivity (see [22] where it is shown that the string tension increases monotonically with the increase of $\left.m_{H} / m_{\gamma}\right)$.

At $\mu_{D}=0$ the extended $\mathcal{N}=2$ supersymmetry is restored and the string becomes BPS saturated reaching the bound $T=2 \pi \eta$, see Sec. 4 . Let us emphasize that $2 \pi \eta$ is the lower bound only when $\mu_{D}=0$. Note, that the number of states in the string supermultiplet still does not jump even at $\mu_{D}=0$. Just the "long" multiplet of $\mathcal{N}=1$ SUSY becomes the "short" multiplet of $\mathcal{N}=2$ SUSY.

Another way to reach the same conclusion is as follows. The type I superconductivity implies that there exist a scalar field with nonvanishing vev whose mass is below that of photon, see Eqs. (65) and (66). The reason for this is that the scalar field with the lowest mass determines the large distance tail of the vortex and ensures the attraction between different vortices. For large $\mu_{D}$ the field $\varphi$ which form the string solution is the eigenvector of the mass matrix with the lowest mass (70).

Let us consider now the region of small $\mu_{D}$ and show that the scalar fields which form the string solution have a non-zero projection on the state with the lowest mass. To the leading order in $\omega$ two normalized eigenvectors of the mass matrix (62) with the lowest eigenvalue $m_{\gamma}^{2}(1-\omega)$ are

$$
\frac{1}{2}\left(\begin{array}{c}
1 \\
1 \\
0 \\
0 \\
-\sqrt{2} \\
0
\end{array}\right), \quad \frac{1}{2}\left(\begin{array}{c}
0 \\
0 \\
1 \\
1 \\
0 \\
-\sqrt{2}
\end{array}\right) .
$$

Let us call by Higgs field the combination of scalars which acquire non-zero vev at infinity. It is proportional to the vector

$$
\frac{1}{\sqrt{2}}\left(\begin{array}{c}
1 \\
1 \\
0 \\
0 \\
0 \\
0
\end{array}\right),
$$

see (61). On the string solution this field changes from zero at the origin to its vev $\xi$ at infinity. Now it is easy to see that this vector has a non-zero projection on the first eigenvector in Eq. (83).

\section{Conclusions}

In this paper we studied ANO vortices near the monopole point in the Seiberg-Witten theory perturbed by the mass of the adjoint matter. This perturbation reduces to FI 
$F$-term as well as to the mass term of the $A_{D}$ field within the QED effective description. We showed explicitly that FI term does not break $\mathcal{N}=2$ supersymmetry and the ANO string is BPS-saturated if the mass parameter $\mu_{D}=0$. Then we break $\mathcal{N}=2$ SUSY by non-zero $\mu_{D}$ and show that the superconductivity at the monopole point is of the type I.

Note, that although we referred to the particular perturbation by the adjoint mass, our results are valid for a generic perturbation. Indeed, for any perturbation one expand the effective superpotential near the monopole points in powers of $a_{D}$. The crucial role is played by a linear term (generalized FI term) which ensures the monopole condensate. It is clear that an appearance of linear term is generic, to have it zero one needs a very special perturbation. The string is BPS in the linear approximation. Then the next quadratic term breaks $\mathcal{N}=2$, so the corresponding central charge seizes to exist.

In the broken $\mathcal{N}=2 \mathrm{QED}$, which corresponds to the type I superconductor, the ANO string tension satisfy the upper bound (82). In particularly, in the limit of large $\mu_{D}$ the tension is $2 \pi \eta / \log \omega$, see Eq. (81). This means that vortices are bound by attractive forces. In particular, the infinite towers of "exotic" hadron states corresponding to all integer winding numbers $n$ of strings emerge in the hadron spectrum.

Of course, if we increase $\mu$ and approach $\mu \sim \Lambda$ the unwanted strings with $n>1$ become broken by the $W$-boson creation. Moreover, as it is shown in [9] within the brane approach the unwanted $\left(N_{c}+1\right) / 2$ multiplicity of the spectrum in $S U\left(N_{c}\right)$ theory also disappears at $\mu \sim \Lambda$, and quark can be connected with antiquark by only one string. This string is believed to be responsible for the confinement in the SQCD in the limit of large $\mu$ [23].

However, this brane picture is hard to implement in the field theory. One reason for this is that at large $\mu$ dual QED enters the strong coupling regime. Another one is probably even more fundamental. The point is that the role of matter fields in the effective QED (四) is played by monopoles. As $\mu$ approaches $\Lambda$ the inverse mass of the dual photon (24) approaches the size of monopole (which is of order of inverse $W$ boson mass, $\left.1 / m_{W} \sim 1 / \Lambda\right)$. Under these conditions we hardly can consider monopoles as local degrees of freedom and the dual QED effective description breaks down. In particular, we do not have a field-theoretical description of $n=1$ string in the region of large $\mu \geq \Lambda$.

On the other hand, at small $\mu$ we have weak coupling QED description, strings and confinement. However, we have an infinite tower of unwanted states in the hadron spectrum.

\section{Acknowledgments}

Authors are grateful to Gregory Gabadadze, Amihay Hanany, Mikhail Shifman and Matthew Strassler for helpful discussions. We are thankful to the Institute for Theoretical Physics at Santa Barbara, where this work was initiated, for the support during 
the SUSY99 program provided by the NSF grant PHY 94-07194. A. Y. would like to thank the Theoretical Physics Institute at the University of Minnesota for hospitality and support. The work of A. V. is supported in part by DOE under the grant DEFG02-94ER40823, A. Y. is partially supported by the Russian Foundation for Basic Researches under grant No. 99-02-16576 and by the US Civilian Research and Development Foundation under grant No. RP1-2108.

\section{References}

[1] S. Mandelstam, Phys. Rep. 23C 145 (1976);

G. t' Hooft, in Proceed. of the Europ. Phys. Soc. 1975, ed. by A. Zichichi (Editrice Compositori, Bologna, 1976) p. 1225.

[2] A. A. Abrikosov, Sov. Phys. JETP, 321442 (1957);

H. B. Nielsen and P. Olesen, Nucl. Phys. B61 45 (1973).

[3] N. Seiberg and E. Witten, Nucl. Phys. B426, 19 (1994) hep-th/9407087.

[4] N. Seiberg and E. Witten, Nucl. Phys. B431, 484 (1994) hep-th/9408099.

[5] S. Elitzur, A. Forge, A. Giveon and E. Rabinovici, Phys. Lett. B353, 79 (1995) hep-th/9504080; Nucl. Phys. B459, 160 (1996) hep-th/9509130.

S. Elitzur, A. Forge, A. Giveon, K. Intriligator and E. Rabinovici, Phys. Lett. B379, 121 (1996) hep-th/9603051.

[6] T. J. Hollowood, V. V. Khoze, W. Lee and M. P. Mattis, Nucl. Phys. B570, 241 (2000) hep-th/9904116.

[7] A. Gorsky, A. Vainshtein and A. Yung, Nucl. Phys. B584, 197 (2000) [hepth/0004087.

[8] M. R. Douglas and S. H. Shenker, Nucl. Phys. B447, 271 (1995) hep-th/9503163].

[9] A. Hanany, M. J. Strassler and A. Zaffaroni, Nucl. Phys. B513, 87 (1998) [hepth/9707244.

[10] M. Strassler, Prog. Theor. Phys. Suppl. 131439 (1998) hep-lat/9803009.

[11] W. G. Fuertes and J. M. Guilarte, Phys. Lett. B437, 82 (1998) hep-th/9807218; J. D. Edelstein, W. G. Fuertes, J. Mas and J. M. Guilarte, Phys. Rev. D62, 065008 (2000) hep-th/0001184.

[12] P. Fayet and J. Iliopoulos, Phys. Lett. B51, 461 (1974).

[13] Z. Hlousek and D. Spector, Nucl. Phys. B370, 143 (1992).

J. Edelstein, C. Nunez and F. Schaposnik, Phys. Lett. B329, 39 (1994) [hepth/9311055. 
[14] A. Kapustin and M. J. Strassler, JHEP 9904, 021 (1999) [hep-th/9902033.

[15] A. Gorsky and M. Shifman, Phys. Rev. D61, 085001 (2000) hep-th/9909015.

[16] S. C. Davis, A. Davis and M. Trodden, Phys. Lett. B405, 257 (1997) [hepph/9702360.

[17] P. G. De Gennes, Superconductivity of Metals and Alloys, Benjamin, New York, 1966.

[18] E. B. Bogomolny, Sov. J. Nucl. Phys. 24449 (1976).

[19] X. Hou, Abrikosov String in $\mathcal{N}=2$ Supersymmetric QED, hep-th/0005119.

[20] D. Kutasov, A. Schwimmer and N. Seiberg, Nucl. Phys. B459, 455 (1996) [hepth/9510222.

[21] A. Yung, Nucl. Phys. B562, 191 (1999) hep-th/9906243].

[22] E. B. Bogomolny and A. I. Vainshtein, Sov. J. Nucl. Phys. 23588 (1976).

[23] E. Witten, Nucl. Phys. B507, 658 (1997) hep-th/9706109. 